\title{
Intracardiac Echo Imaging in Atrial and Ventricular Arrhythmia Ablation
}

Editors

PASQUALE SANTANGELI

FERMIN C. GARCIA

LUIS C. SÁENZ

CARDIAC

ELECTROPHYSIOLOGY

CLINICS

www.cardiacEP.theclinics.com

Consulting Editors

RANJAN K. THAKUR

ANDREA NATALE

June 2021 • Volume 13 - Number 2 